\title{
Prophylactic Use of Ganoderma lucidum Extract May Inhibit Mycobacterium tuberculosis Replication in a New Mouse Model of Spontaneous Latent Tuberculosis Infection
}

\author{
Lingjun Zhan ${ }^{\dagger}$, Jun Tang ${ }^{\dagger}$, Shuzhu Lin, Yanfeng Xu, Yuhuan Xu and Chuan Qin * \\ Key Laboratory of Human Diseases and Comparative Medicine, Ministry of Health, Institute of Laboratory Animal Science, \\ Peking Union Medical College, Chinese Academy of Medical Sciences and Comparative Medicine Center, Beijing, China
}

\section{OPEN ACCESS}

Edited by:

Andres M. Perez,

University of Minnesota, USA

Reviewed by:

Michelle Martinez-Montemayor, Universidad Central del Caribe,

Puerto Rico

Paras Jain,

Albert Einstein College of Medicine,

USA

*Correspondence:

Chuan Qin

qinchuan@pumc.edu.cn

${ }^{\dagger}$ Co-first authors.

Specialty section: This article was submitted to

Infectious Diseases,

a section of the journal

Frontiers in Microbiology

Received: 14 May 2015 Accepted: 10 December 2015 Published: 08 January 2016

Citation:

Zhan L, Tang J, Lin S, Xu Y, Xu Y and Qin C (2016) Prophylactic Use of Ganoderma lucidum Extract May Inhibit Mycobacterium tuberculosis Replication in a New Mouse Model of Spontaneous Latent Tuberculosis Infection. Front. Microbiol. 6:1490. doi: 10.3389/fmicb.2015.01490
A mouse model of spontaneous latent tuberculosis infection (LTBI) that mimics LTBI in humans is valuable for drug/vaccine development and the study of tuberculosis. However, most LTBI mouse models require interventions, and a spontaneous LTBI mouse model with a low bacterial load is difficult to establish. In this study, mice were IV-inoculated with 100 CFU Mycobacterium tuberculosis H37Rv, and a persistent LTBI was established with low bacterial loads $\left(0.5 \sim 1.5 \log _{10}\right.$ CFU in the lung; $<4 \log _{10}$ CFU in the spleen). Histopathological changes in the lung and spleen were mild during the first 20 weeks post-inoculation. The model was used to demonstrate the comparative effects of prophylactic and therapeutic administration of Ganoderma lucidum extract (spores and spores lipid) in preventing H37Rv replication in both lung and spleen. H37Rv was inhibited with prophylactic use of $\mathrm{G}$. Iucidum extract relative to that of the untreated control and therapy groups, and observed in the spleen and lung as early as post-inoculation week 3 and week 5 respectively. H37Rv infection in the therapy group was comparable to that of the untreated control mice. No significant mitigation of pathological changes was observed in either the prophylactic or therapeutic group. Our results suggest that this new LTBI mouse model is an efficient tool of testing anti-tuberculosis drug, the use of G. Iucidum extract prior to $M$. tuberculosis infection may protect the host against bacterial replication to some extent.

Keywords: mouse model, spontaneous latent tuberculosis infection, Ganoderma lucidum extract, prophylactic use, inhibited Mycobacterium tuberculosis replication

\section{INTRODUCTION}

Approximately one third of the world's population is infected with Mycobacterium tuberculosis, and about $90 \%$ of these infections are latent. A better understanding of mechanisms leading to latency is required to help prevent, control, treat, and eliminate tuberculosis infection and disease.

Similar to other latent infections, M. tuberculosis in latent tuberculosis infection (LTBI) replicates at low levels in the infected organs, and therefore histopathology results 
are absent or mild. There are several established LTBI animal models, including mice (Scanga et al., 1999), guinea pigs (Kashino et al., 2008), rats (Singhal et al., 2011), rabbits (Manabe et al., 2008), and nonhuman primates (Lin et al., 2009a). These models have been used to identify host factors that contribute to the establishment and maintenance of M. tuberculosis latency, and reactivation of replication. One of the most recognized LTBI mouse models is the Cornell model, which is created by inhibiting $M$. tuberculosis replication with intervening factors (Lenaerts et al., 2004; Woolhiser et al., 2007). However, there is no currently available mouse model of spontaneously paucibacillary tuberculosis.

The three primary parameters used for evaluating vaccine or drug candidate against tuberculosis are the bacterial load, pathological change in the lung, and the tuberculosis relapse rate in the latter phase of LTBI. With the current LTBI mouse model, the period of latency is relatively long, and to complete the evaluation can require 3-7 months (Ziv et al., 2001; Ha et al., 2003; Zhang et al., 2011). Thus, the current models are inefficient and costly, and slow the development of new tuberculosis vaccines and drug treatments.

A previous study showed that a proteoglycan extracted from the fruiting bodies of the bracket (polypore) fungus G. lucidum could be a preventative of diabetic complications (Pan et al., 2013). Furthermore, triterpenes of G. lucidum were shown to exert anti-lung cancer activity in vitro and in vivo, and such anticancer activity was mediated by enhancing immunomodulation and induction of cellular apoptosis (Feng et al., 2013). Also of note is that polysaccharides purified from the submerged culture of G. formosanum can activate macrophages and protect mice against Listeria monocytogenes infection (Wang et al., 2011). G. lucidum can also regulate natural killer cells (Chien et al., 2004), macrophages (Yeh et al., 2010), T cells (Lai et al., 2010; Yoshida et al., 2012), and dendritic cells (Meng et al., 2011), all of which are actively involved in the innate and adaptive immune responses to $M$. tuberculosis infection. Thus, we wondered whether G. lucidum could protect the host from M. tuberculosis infection.

To test our hypothesis, in the present study we established a novel mouse model of spontaneous LTBI, with a shorter latency period and lower bacterial load than previous models. We then evaluated the protective effects against $M$. tuberculosis infection in this new model exerted by the Takayama G. Lucidum (MeiShanTang, Hong Kong), which is rich in triterpenes, and is proven effective in treating simian acquired immune deficiency syndrome via the immune system (Lu et al., 2011).

\section{MATERIALS AND METHODS}

\section{Ethics Statement}

The Institute of Animal Use and Care Committee of the Institute of Laboratory Animal Science, Peking Union Medical College approved all protocols and procedures that involved animals (ILAS-PC-2013-015). All mice were housed in plastic cages (6/cage) with free access to drinking water and a pellet diet, under controlled humidity (50 $\pm 10 \%)$, light (12/12 h light/dark cycle), and temperature $\left(23 \pm 2^{\circ} \mathrm{C}\right)$ conditions in an Animal Biosafety Level 3 (ABSL-3) facility. Prior to procedures performed at each time-point, mice were fasted overnight and then given anesthesia.

\section{Establishment of the LTBI Mouse Model}

\section{Bacterial Strain}

The M. tuberculosis strain H37Rv was first cultured in Löwenstein-Jensen plates (L-J plates) for 3 weeks, to mid$\log$ phase. Then the bacteria were harvested using a sterilized L-shaped glass rod and resuspended in $0.9 \% \mathrm{NaCl}$ in a glass grinder. The suspension was filtered through a $5 \mu \mathrm{m}$ membrane and the bacterial density adjusted to $\sim 1 \times 10^{7}$ colony-forming units $(\mathrm{CFU}) / \mathrm{mL}$. An aliquot of bacilli were then plated on an L-J plate for CFU enumeration.

\section{Mice}

Specific-pathogen free female C57BL/6 mice (6-8 weeks old, $n=450$ ) were obtained from Vital River Laboratory Animal Technology (China). Mice were maintained in an ABSL-3 specific pathogen-free facility and allowed food and water ad libitum.

\section{Inoculation of Mice with M. tuberculosis}

The mice were randomly and equally apportioned to an infection or a blank control group, 150 mice for each experiment, 75 mice for infection and blank control group each, the experiment would be repeated 3 times with 1 week interval. Mice in the infection group were inoculated through the tail vein with $0.1 \mathrm{~mL}$ of $\mathrm{H} 37 \mathrm{Rv}$ $\left(1 \times 10^{3} \mathrm{CFU} / \mathrm{mL}\right)$, that was $100 \mathrm{CFU}$ for each mouse, and control mice were similarly injected with $0.1 \mathrm{~mL} 0.9 \% \mathrm{NaCl}$. All procedures were performed in a biosafety cabinet located in the ABSL-3 facility.

\section{Analysis of Pathologic Changes}

Six mice were sacrificed at each time-point (weeks 1, 3, 5, 8, $12,16,20$, and 24), for the first experiment, the rest mice were sacrificed at week 34, 38, 44 and 52(see Supplementary Image 2), six for each time-point, and for the latter two experiments, the rest mice were sacrificed at 52th week. The lung, spleen, and liver were removed from each animal; gross lesions were examined and described. The necropsy tissues were fixed and paraffin-embedded. The sections were cut and stained with hematoxylin and eosin, and reviewed by a veterinary pathologist. Semi-quantitative scores were assigned to reflect the lesion size and extent of inflammation of the entire lung field:,$+ 25 \%$; ++ , $50 \% ;++, 75 \%$.

\section{Quantitative Culture of Lung and Spleen Homogenates}

The lung and spleen tissues taken at necropsy were washed in $4 \%$ $\mathrm{H}_{2} \mathrm{SO}_{4}$ and homogenized in $0.9 \% \mathrm{NaCl}$. Serial dilutions of the homogenous lung fluid were used to inoculate the L-J medium culture in tubes, which were incubated at $37^{\circ} \mathrm{C}$ and $5 \% \mathrm{CO}_{2}$. The bacterial counts in the tubes were read after 21 days. At each timepoint, the mean of the bacterial loads in each organ type of 6 mice were calculated. 


\section{Evaluation of the Protective Effect of G. Lucidum in the LTBI Mouse Model Study Design}

The mice were divided into an LTBI mouse model control group, a G. lucidum prophylaxis group, and a therapy group. Mice in the control group were inoculated with $M$. tuberculosis as described above, but were fed normally and not given G. lucidum extract. The prophylaxis group received daily doses of G. lucidum mushroom extract (described below) beginning 1 month before M. tuberculosis inoculation and until 16 weeks after inoculation. In the therapy group, mice were given daily doses of G. lucidum mushroom extract beginning at the time of inoculation and lasting 16 weeks.

\section{Preparation of G. Iucidum Extract-Containing Food}

Takaya Shell-broken Ganoderma Lucid Spores and Spores Lipid, which were rich in terpene carbon dioxide extraction from included species of G. lucidum (MeiShanTang, Hong Kong) were mixed with the mouse feed powder and water, and then the mixture was manually formulated into $\sim 15-\mathrm{cm}$-long feed strips with diameter of $1.5 \mathrm{~cm}$. The strips were dried in the $37^{\circ} \mathrm{C}$ oven for $48 \mathrm{~h}$. Each mouse received daily $15 \mathrm{mg}$ of $G$. lucidum spores and $15 \mathrm{mg}$ spore lipids in $4 \mathrm{~g}$ of feed (Lu et al., 2011).

\section{Mouse Trails}

In the prophylaxis group, the feeding duration of G. lucidum was 20 weeks, starting 1 month before $M$. tuberculosis inoculation and continuing to the 16th week post-inoculation. In the therapy group, dietary G. lucidum (including spores and spore lipids) was administrated for 16 weeks, from the inoculation of $M$. tuberculosis to the 16th week postinoculation. The effect of G. lucidum on $M$. tuberculosis infection in mice was evaluated according to the bacterial load in the lung and spleen, and the pathological changes in the lung, spleen, and liver at 3, 5, 8, and 16 weeks post-inoculation.

\section{Fluorescence-Activated Cell Sorting (FACS) Analysis of Immune Cells}

Dendritic, natural killer, $\mathrm{CD} 4^{+} / \mathrm{CD}^{+} \mathrm{T}$ cells, and regulatory $\mathrm{T}$ (Treg) cells in the peripheral blood and lung were stained with specific antibody (all from eBioscience) for $30 \mathrm{~min}$ at $4^{\circ} \mathrm{C}$. The following mouse antibodies were used: CD11b-fluorescein isothiocyanate (FITC) and CD4-FITC; I-AB-phycoerythrin (PE), CD86-PE, CD8-PE, CD49b-PE, and FOXP3-PE; CD80-peridinin chlorophyll protein complex (PERCP) and CD3-PERCP; and CD11c-allophycocyanin (APC), perforin-APC, and CD25-APC. The stained cells were analyzed by FACS.

\section{Statistical Analyses}

Quantitative data are expressed as the mean \pm standard error of the mean (SEM), and analyzed using a two-tailed Student's $t$-test and ANOVA. $P<0.05$ was considered statistically significant.

\section{RESULTS}

\section{Bacterial Loads in the Lung and Spleen of the LTBI Mouse}

The bacterial loads of lung and spleen in the infection and blank control groups at $1,3,5,8,12,16,20$, and 24 weeks after inoculation with $M$. tuberculosis was determined (Figures 1A,B). No M. tuberculosis was detected in the blank control mice at any time-point. In the lungs of the infection group, the mean bacterial load was $\sim 1.5 \log _{10} \mathrm{CFU}$ at 3 rd week, at the lowest level found $\left(\sim 0.5 \log _{10} \mathrm{CFU}\right)$ at 8 th week, fluctuated from indeterminably low to $\sim 2 \log _{10} \mathrm{CFU}$ at $8-20$ th week, and was $\sim 2.5 \log _{10} \mathrm{CFU}$ at week 24 (Figure 1A). In the spleens of the mice in the infection group, the mean bacterial load at 3 rd week was $4.5 \log _{10}$ CFU, then $2.1 \log _{10} \mathrm{CFU}$ at 8 th week, and was subsequently progressively higher at each time-point, to $>4 \log _{10} \mathrm{CFU}$ at week 24 (Figure 1B). Compared with blank control, both the bacteria loads of spleen and lung in infected group had significant statistic difference $\left({ }^{* * *} P<0.001\right)$.

There were no observed gross pathological changes throughout the infection course in any group, but histopathological changes were observed under the light microscope. In the lung at week 3, mild infiltration with inflammatory cells in the peripheral vascular was observed in the lung. At week 5, a granuloma-like structure in the lung was observed, and scored as a severe lesion $(+++)$. However, at week 8, the histopathologic lesion was not seen, and only a few inflammatory cells infiltrated vessels. Infiltration of inflammatory cells in the lung was greater at week 16 (Figure 2).

In the spleen at $3 \mathrm{rd}$ week, several small granulomas (diameter, $5-50 \mu \mathrm{m}$ ) were detected in the white pulp. At week 5, the granulomas in the white pulp of the spleen were of diameters $20-150 \mu \mathrm{m}$, but at week 8 granuloma sizes were $5-20 \mu \mathrm{m}$. At week 20, several granuloma-like lesions of size 10-50 $\mu \mathrm{m}$ were observed in the spleen white pulp (Figure 2).

At week 3-16, granuloma-like lesions were occasionally observed in the hepatic lobule. The size of irregularly shaped granuloma-like lesions at week 3 was $\sim 50 \times 350 \mu \mathrm{m}$, and smaller granuloma-like lesions $(20-50 \mu \mathrm{m})$ were present from weeks 5 to 20 (Figure 2).

\section{Bacterial Loads in the Lung and Spleen of G. Iucidum Extract-Treated Mice}

The condition of mice inoculated with $100 \mathrm{CFU}$ M. tuberculosis resembled LTBI during the first 20 weeks post-inoculation. Therefore, to evaluate the effect of the G. lucidum extract, we determined the bacterial loads of the control and model mice at time-points up to 16 weeks post-inoculation.

In the prophylaxis group, in the lung the bacterial load was essentially none up through week 8 , but was $(0.42 \pm 0.34)$ $\log _{10} \mathrm{CFU}$ at week 16 (Figure 3A). In the therapy group, the $M$. tuberculosis level in the lung remained essentially undetectable through the first 3 weeks, but was $(1.39 \pm 0.77) \log _{10}$ CFU at week 5 , $(0.42 \pm 0.42) \log _{10} \mathrm{CFU}$ at week 8 , and again undetectable at week 16. In the spleens of the prophylaxis group, the mean 

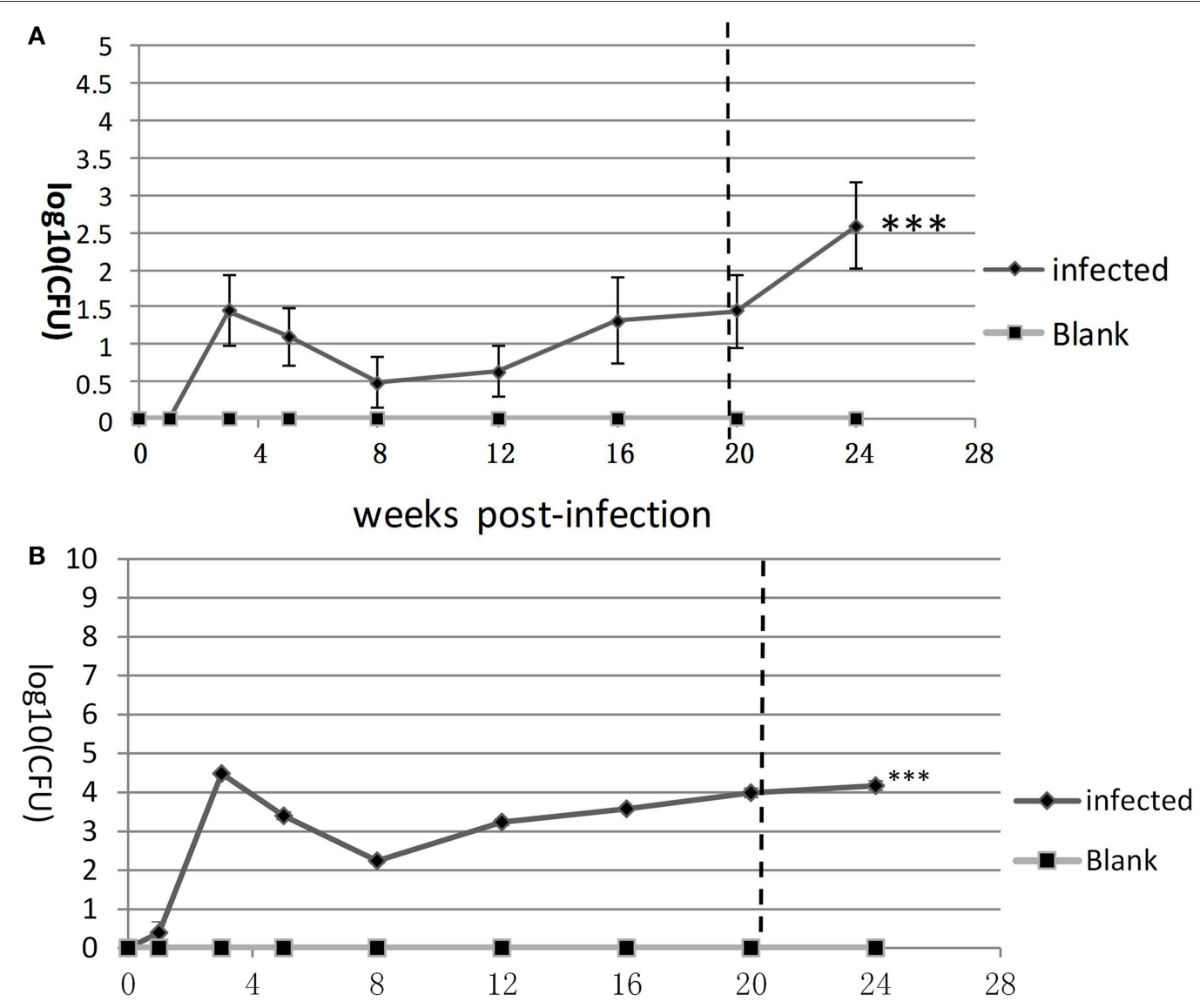

Weeks post-infection

FIGURE 1 | The replication kinetics of $\boldsymbol{M}$. tuberculosis in the lung and spleen. Bacterial loads $\left(\log _{10} \mathrm{CFU}\right)$ in (A) the lung and (B) spleen were counted on the L-J medium from week 1 to week 24 . Six mice for each time-point in each group. One of three similar experiments is shown. The SEMs are plotted as error bars. $T$-test and ANOVA analysis, ${ }^{\star * \star} P<0.001$.

M. tuberculosis levels were $(1.69 \pm 0.71) \log _{10}$ CFU at week 3, (1.12 \pm 0.77$) \log _{10} \mathrm{CFU}$ at week $5,(3.48 \pm 0.11) \log _{10} \mathrm{CFU}$ at week 8 , and $(2.41 \pm 0.41) \log _{10}$ CFU at week 16 . In the spleens of the therapy group, the bacterial loads were similar to that of the control group: a peak value at $(4.33 \pm 0.75) \log _{10} \mathrm{CFU}$ at week $3,(3.56 \pm 0.05) \log _{10} \mathrm{CFU}$ at week $5,(3.22 \pm 0.17) \log _{10} \mathrm{CFU}$ at week 8 , and (3.04 \pm 0.17$) \log _{10} \mathrm{CFU}$ at week 16(Figure 3B). As for spleen bacteria loads, the prophylaxis group showed statistical differences at both week 3 and 5, compared with both blank control and therapy group (W3, $P<0.05$; W5, $P<0.01$ ), and only with control group at week $8(P<0.05)$; but for lung bacteria loads, the prophylaxis group revealed significant statistic difference only at week 5 , compared with therapy group (Figures 3A,B).

\section{Histopathologic Changes in G. lucidum-Treated Mice}

There were no macroscopic changes in the lungs, spleens, or livers in any of the groups, and no visible differences in the microscopic histology of the spleens or livers. However, histological differences in the lung were detected.

In the LTBI model control (untreated) group, inflammatory lesions were aggravated at week 5 compared with week 3 , but were not seen from week 8 to 16 . Similar to the control group, in the prophylaxis and therapy group small lesions peaked at week 5, and then were absent at week 8-16. At week 5, inflammation in the prophylaxis group was milder than inflammation in the control group (Figure 4A). Compared with the control group, mice in the G. lucidum prophylaxis group had lower pathology scores during the infection course, but the difference was not significant $(P>0.05$; Figure 4B).

\section{Percentages of Dendritic Cell, Natural Killer Cell, and Treg Cell, and the Ratios of $\mathrm{CD}^{+} / \mathrm{CD}^{+} \mathrm{T}$ cell in Blood}

The number of dendritic cell, natural killer cell, Treg cell, and the ratio of $\mathrm{CD}^{+} / \mathrm{CD} 8^{+} \mathrm{T}$ cell in the peripheral blood 


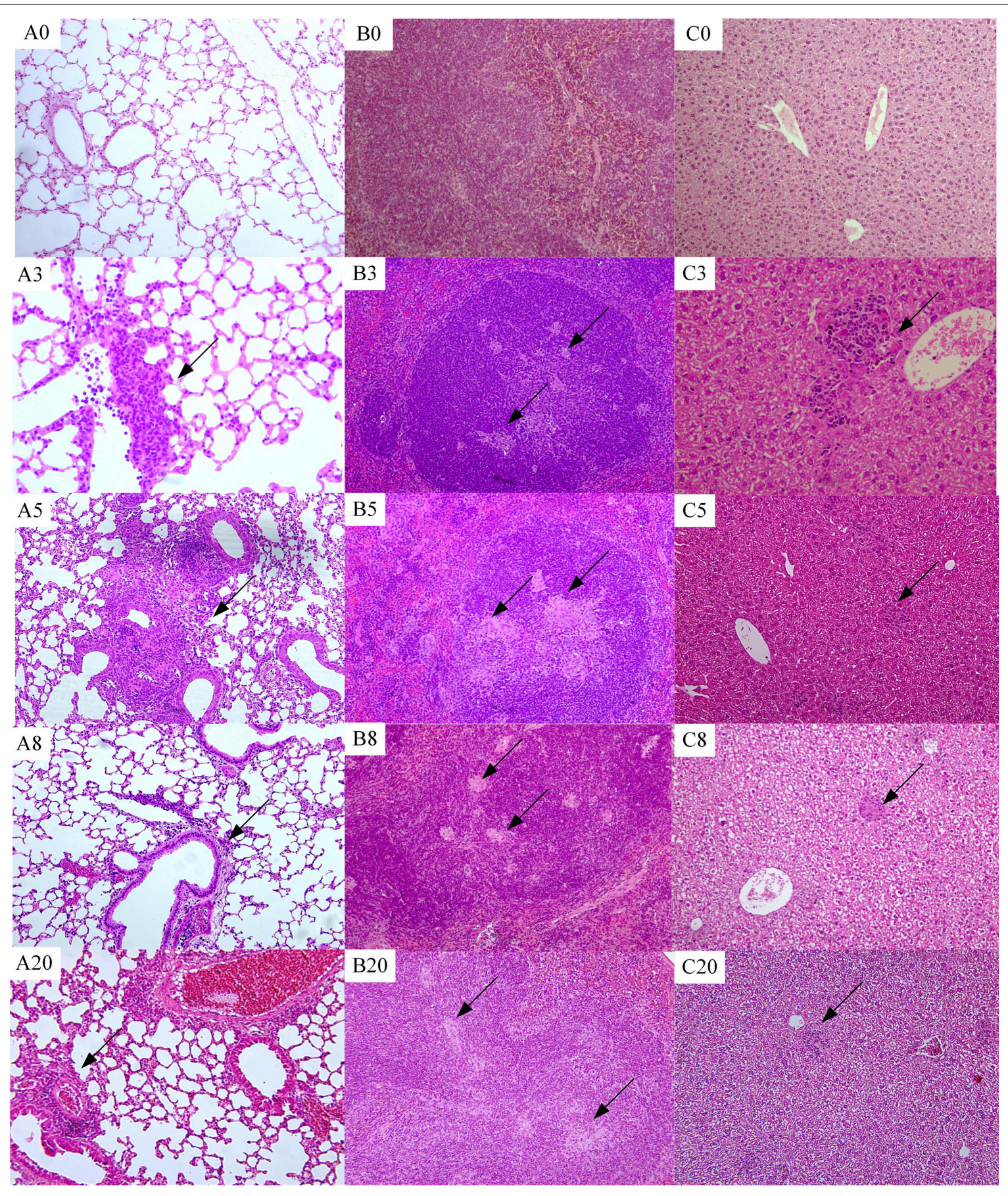

FIGURE 2 | Histopathological changes over time (100x). (AO-A20) Lung histology at weeks 0, 3, 5, 8, and 20, respectively. (B0-B20) Spleen histopathology at weeks $0,3,5,8$, and 20. (C0-C20) Liver histopathology at weeks 0, 3, 5, 8, and 20. Arrow indicates granulomas-like lesion.

were analyzed by FACS (Figure 5). In the prophylaxis group, the percentages of dendritic cell were $14.8 \pm 5.04 \%$ at week $3,6.56 \pm 1.93 \%$ at week $5,1.22 \pm 0.10 \%$ at week 8 , and $7.64 \pm 2.69 \%$ at week 16 . In the therapy and control group, the percentages of dendritic cells were similar to that of the prophylaxis group, progressively increasing over time from $9.24 \pm 1.55 \%$ at week 3 to $18.48 \pm 2.00 \%$ at week 16 .

The percentages of natural killer cell in the prophylaxis group was $2.70 \pm 0.68 \%$ at week $3,9.82 \pm 1.48 \%$ at week $5,15.76 \pm$ $2.63 \%$ at week 8 , and $7.15 \pm 1.20 \%$ at week 16 . The percentages of natural killer cell in the control and therapy group were 

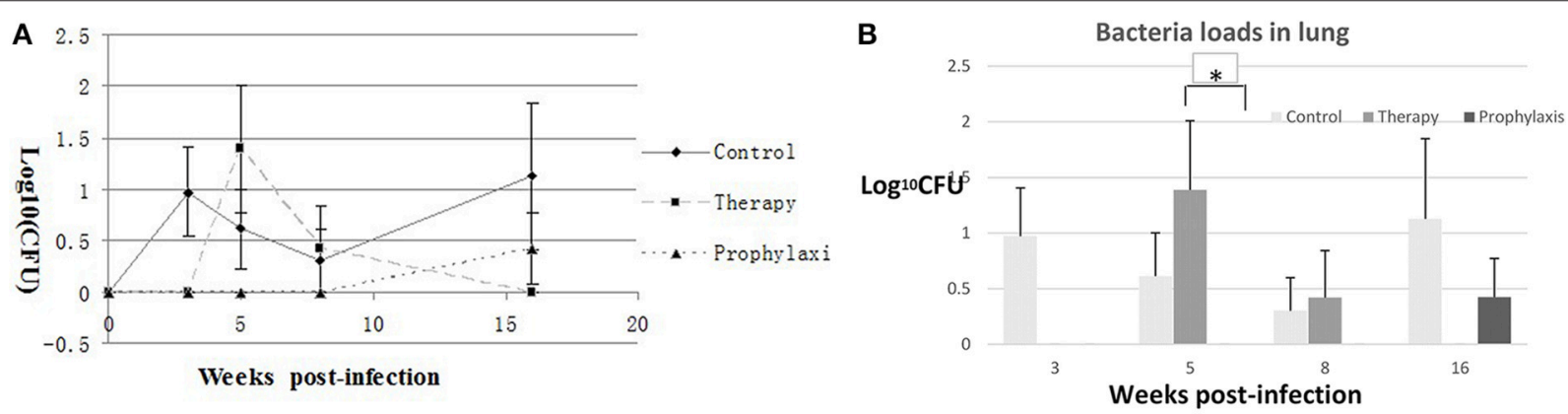

Bacteria loads in spleen
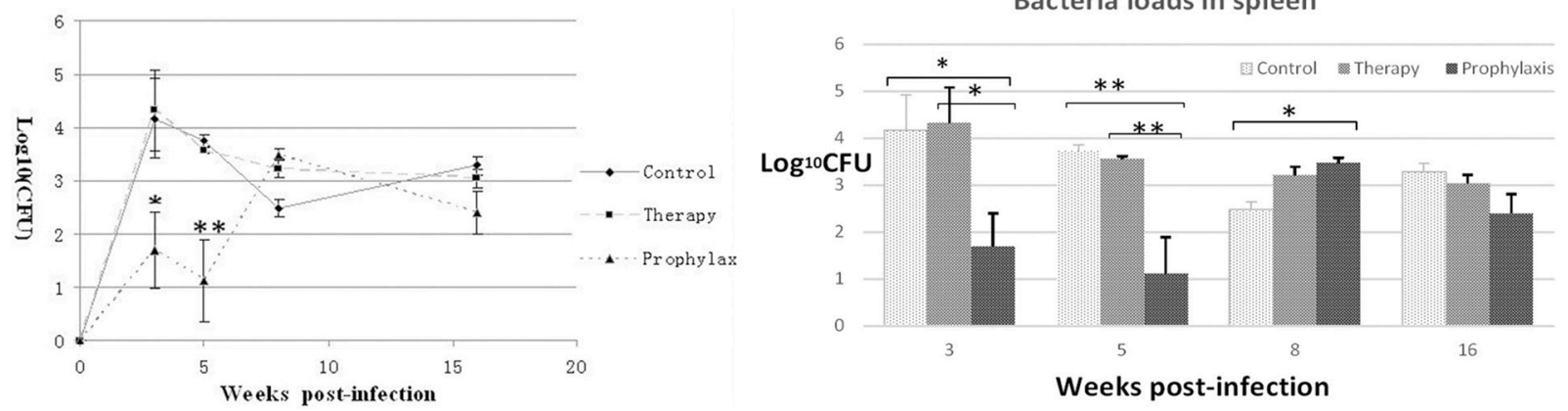

FIGURE 3 | Differences in bacterial loads between the control group and G. lucidum-treated (therapy and prophylaxis) mice. Bacterial loads (log 10 CFU) in the (A) lung and (B) spleen were counted on the L-J medium from week 0 to week 16. One of three similar experiments is shown. The SEMs are plotted as error bars. ANOVA analysis, ${ }^{\star} P<0.05,{ }^{*} P<0.01$.

comparable: $\sim 2, \sim 10, \sim 5$, and $\sim 7 \%$ at weeks $3,5,8$, and 16 , respectively.

The ratios of $\mathrm{CD} 4^{+} / \mathrm{CD}^{+} \mathrm{T}$ cells in all three groups were similar and stable at $\sim 1.8$ throughout the infection period, except for the prophylaxis group, in which a mean ratio of $\sim 4.2$ was observed at week 8 .

The percentages of Treg cells in the prophylaxis group were $0.86 \pm 0.05 \%$ at week $3,0.49 \pm 0.08 \%$ at week $5,1.63 \pm 0.08 \%$ at week 8 , and $0.76 \pm 0.12 \%$ at week 16 . In the control and therapy group, the percentages of Treg cells were similar $(\sim 1 \%)$ at week 5 , and progressively and similarly decreased from week 5 to 16 (Figure 5).

\section{DISCUSSION}

We present here a novel mouse model of spontaneous LTBI, which closely resembles human LTBI. The model was generated through experimental inoculation of $M$. tuberculosis without resorting to other means such as anti-tuberculosis drugs treatment or Mycobacterium bovis Bacillus Calmette-Guerin vaccination. A useful application of this model is to evaluate anti-tuberculosis drugs or vaccines during the early latency phase, which would be more efficient than the previous models (Ziv et al., 2001; Ha et al., 2003; Zhang et al., 2011). In the present study, we demonstrated the efficiency of the new mouse LTBI model by testing the effect of G. lucidum extract on $M$. tuberculosis infection, by inhibiting M. tuberculosis replication.
To initiate the LTBI model, C57BL/6 mice were IV-injected with $100 \mathrm{CFU}$ M. tuberculosis inoculum. M. Tuberculosis initially replicated in the lung and spleen, with bacterial loads rising until week 3 , after which infection entered into a latent phase with $M$. tuberculosis levels declining from week 3 and stabilizing at a lower level till week 20. In accord with the bacterial load findings, pathological changes were detected at week 3 , which became prominent at week 5 , and then subsided to only mild perivascular inflammation from week 8 to 20. $M$. tuberculosis replication shifted from relatively active to dormant, resulting in lower bacterial loads and mild pathological changes in both the lung and spleen from weeks 8 to 20 post-inoculation. Thus, the period within 8 weeks would be regarded as "pre-latency phase," and the period from week 8 to week 20 comprised the real latency phase of $M$. tuberculosis infection in this model (Figures 2, 3). The bacteria load curves of spleen and lung were similar to others study, but with lower value and shorter latency period, which would be more efficient in drug evaluation.

Treatment of mice with G. lucidum extract beginning 1 month before inoculation with $M$. tuberculosis (the prophylaxis group) inhibited replication of the bacterium in the lung and spleen during the first 8 weeks postinoculation, relative to untreated control mice and mice treated from the time of inoculation (the therapy group). This inhibition was especially evident in the spleen 5 weeks after inoculation. No significant inhibition of $M$. tuberculosis replication was observed in the therapy group, suggesting 

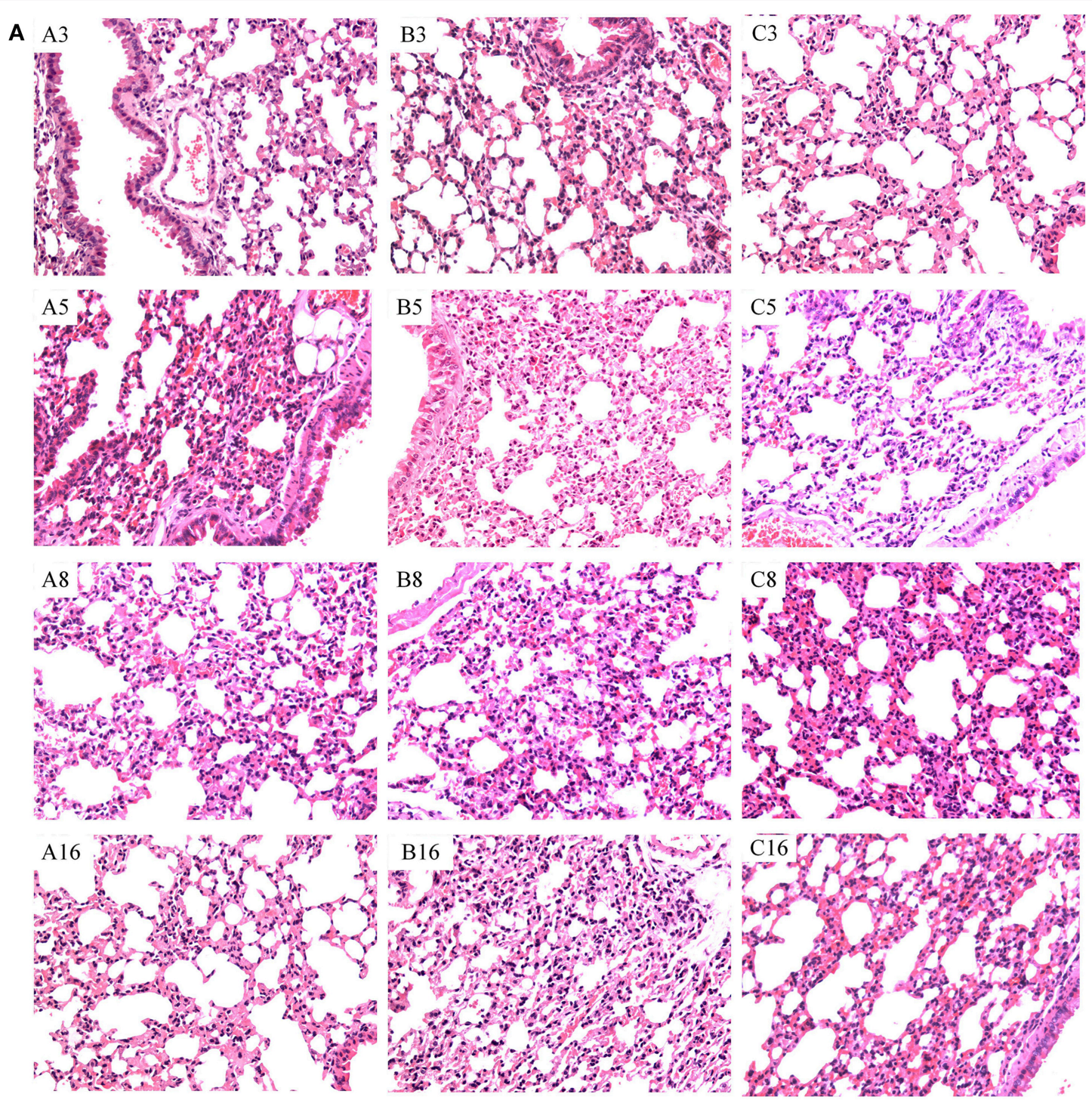

B

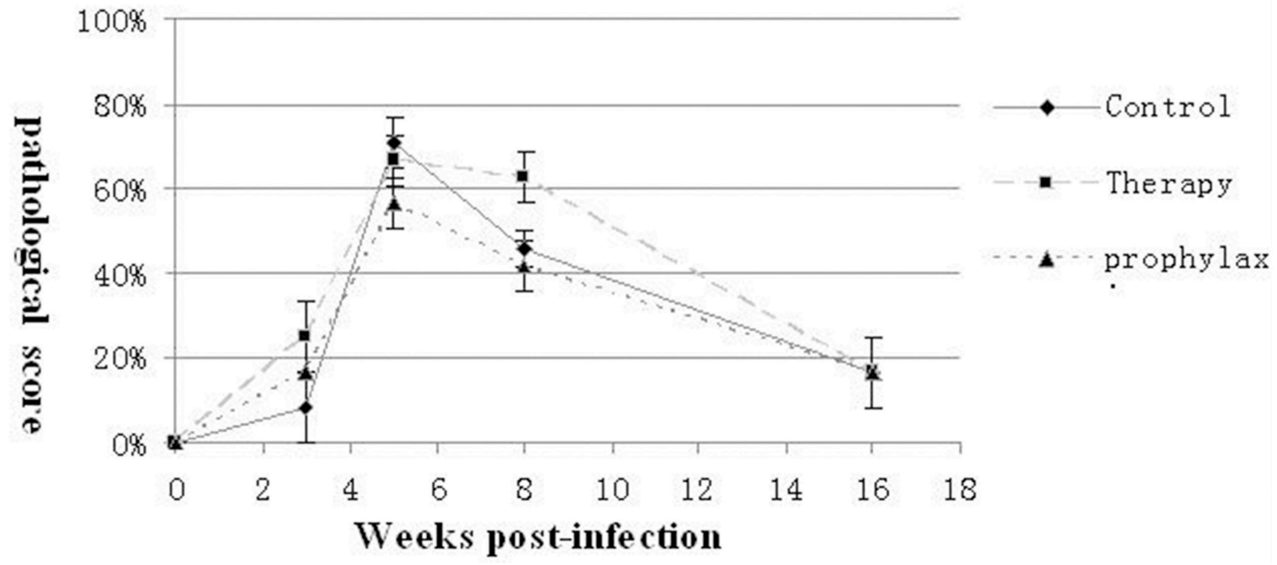

FIGURE 4 | Pathology of the lung. (A) Histopathology of the lungs at weeks 3, 5, 8, and 16 weeks of representative mice in the (A3-A16) control, (B3-B16) prophylaxis, and (C3-C16) therapy groups (200x). (B) Pathological lesion scores in the lung of mice in the control, therapy and prophylaxis groups at weeks $3,5,8$, and 16. SEMs are plotted $(P>0.05)$. 

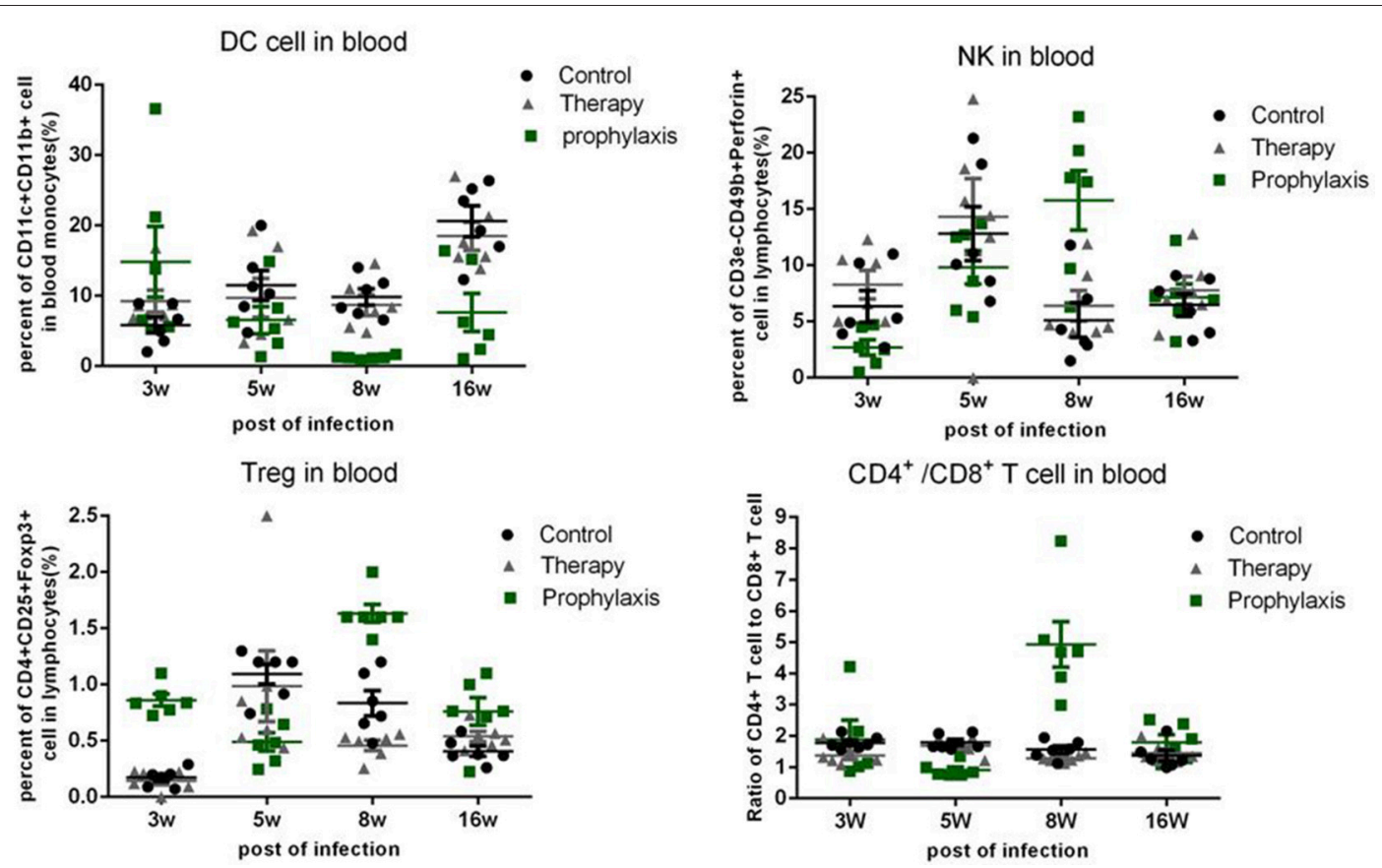

FIGURE 5 | Percentages of dendritic cells (DC), natural killer (NK) cells, and Treg cells, and the ratios of CD4+/CD8 ${ }^{+}$T cells in the blood. The results are expressed as mean \pm SEM, plotted using GraphPad Prism version 4.01 (GraphPad, San Diego, CA).

that early use of G. lucidum extract is probably required for exerting anti- tuberculosis activity in this mouse model (Figure 4).

The changes of immune cell percentages in the peripheral blood did not correlate with the bacterial loads of $M$. tuberculosis or pathological changes observed in the lung and spleen. However, responses of the peripheral blood and lung to inoculation were opposed, with regard to percentages of dendritic cells. At post-inoculation week 3, the number of peripheral dendritic cells in prophylaxis group was higher than that in the control group, but at week 5 was lower than the control group, while the number of dendritic cells in the prophylaxis group lung was higher at week 5 than week 3 (Supplementary Data), and higher than the control group. These changes suggest that dendritic cells may have transferred from the peripheral blood to the lung from week 3 to week 5, and the accumulation of dendritic cells in the lung might participate in and augment the repression of $M$. tuberculosis replication, inferred from the immune effect of G. lucidum on dendritic cells in previous research (Figure 5) (Lin et al., 2009b; Meng et al., 2011).

However, the immune mechanism of G. lucidum extract could not be discerned, and nor if the effective constituent was terpenes, which should be investigated in a further study.

In conclusion, we established a mouse model resembling human $M$. tuberculosis LTBI. The early acute phase of infection (from inoculation to week 8) and the relatively short latency period makes this model useful for evaluating anti-tuberculosis drugs. We utilized the model to investigate the effect of $G$. lucidum extract on $M$. tuberculosis infection. We found that administration of Takaya G. lucidum extract prior to inoculation of $M$. tuberculosis was associated with lower levels of bacterial replication in this model.

\section{AUTHOR CONTRIBUTIONS}

LZ and CQ conceived the concept and designed the experiment. LZ, JT, and SL conducted all the experiments except for the pathological analysis. YX and YX performed the pathology examinations. YL provided the Takaya Ganoderma lucidum extract. LZ performed the data analysis and wrote the manuscript. All the authors read and approved this manuscript for publication.

\section{ACKNOWLEDGMENTS}

This study was supported by the National Science and Technology Major Projects of Infectious Disease (2012ZX10004501-001-005).

\section{SUPPLEMENTARY MATERIAL}

The Supplementary Material for this article can be found online at: http://journal.frontiersin.org/article/10.3389/fmicb. 2015.01490 


\section{REFERENCES}

Chien, C. M., Cheng, J. L., Chang, W. T., Tien, M. H., Tsao, C. M., Chang, Y. H., et al. (2004). Polysaccharides of Ganoderma lucidum alter cell immunophenotypic expression and enhance CD56+ NK-cell cytotoxicity in cord blood. Bioorg. Med. Chem. 12, 5603-5609. doi: 10.1016/j.bmc.2004.08.004

Feng, L., Yuan, L., Du, M., Chen, Y., Zhang, M. H., Gu, J. F., et al. (2013). Anti-lung cancer activity through enhancement of immunomodulation and induction of cell apoptosis of total triterpenes extracted from Ganoderma luncidum (Leyss. ex Fr.) Karst. Molecules 18, 9966-9981. doi: 10.3390/molecules18089966

Ha, S. J., Jeon, B. Y., Kim, S. C., Kim, D. J., Song, M. K., Sung, Y. C., et al. (2003). Therapeutic effect of DNA vaccines combined with chemotherapy in a latent infection model after aerosol infection of mice with Mycobacterium tuberculosis. Gene Ther. 10, 1592-1599. doi: 10.1038/sj.gt.3302057

Kashino, S. S., Napolitano, D. R., Skobe, Z., and Campos-Neto, A. (2008). Guinea pig model of Mycobacterium tuberculosis latent/dormant infection. Microbes Infect. 10, 1469-1476. doi: 10.1016/j.micinf.2008.08.010

Lai, C. Y., Hung, J. T., Lin, H. H., Yu, A. L., Chen, S. H., Tsai, Y. C., et al. (2010). Immunomodulatory and adjuvant activities of a polysaccharide extract of Ganoderma lucidum in vivo and in vitro. Vaccine 28, 4945-4954. doi: 10.1016/j.vaccine.2010.05.037

Lenaerts, A. J., Chapman, P. L., and Orme, I. M. (2004). Statistical limitations to the Cornell model of latent tuberculosis infection for the study of relapse rates. Tuberculosis (Edinb.) 84, 361-364. doi: 10.1016/j.tube.2004.03.002

Lin, P. L., Rodgers, M., Smith, L., Bigbee, M., Myers, A., Bigbee, C., et al. (2009a). Quantitative comparison of active and latent tuberculosis in the cynomolgus macaque model. Infect. Immun. 77, 4631-4642. doi: 10.1128/IAI.00592-09

Lin, Y. L., Liang, Y. C., Tseng, Y. S., Huang, H. Y., Chou, S. Y., Hseu, R. S., et al. (2009b). An immunomodulatory protein, Ling Zhi-8, induced activation and maturation of human monocyte-derived dendritic cells by the NF-kappaB and MAPK pathways. J. Leukoc. Biol. 86, 877-889. doi: 10.1189/jlb.0708441

Lu, Y. Z., Wu, X. X., Chen, S., Yuan, J., Lai, C. H., Bao, L. L., et al. (2011). Effective of Gondeema lucidium preparation in treating simian aquire immune deficiency syndrome. Acta Acad. Med. Sin. 33, 318-324. doi: 10.3881/j.issn.1000503X.2011.03.021

Manabe, Y. C., Kesavan, A. K., Lopez-Molina, J., Hatem, C. L., Brooks, M., Fujiwara, R., et al. (2008). The aerosol rabbit model of TB latency, reactivation and immune reconstitution inflammatory syndrome. Tuberculosis (Edinb.) 88, 187-196. doi: 10.1016/j.tube.2007.10.006

Meng, J., Hu, X., Shan, F., Hua, H., Lu, C., Wang, E., et al. (2011). Analysis of maturation of murine dendritic cells (DCs) induced by purified Ganoderma lucidum polysaccharides (GLPs). Int. J. Biol. Macromol. 49, 693-699. doi: 10.1016/j.ijbiomac.2011.06.029

Pan, D., Zhang, D., Wu, J., Chen, C., Xu, Z., Yang, H., et al. (2013). Antidiabetic, antihyperlipidemic and antioxidant activities of a novel proteoglycan from
Ganoderma lucidum fruiting bodies on $\mathrm{db} / \mathrm{db}$ mice and the possible mechanism. PLoS ONE 8:e68332. doi: 10.1371/journal.pone.0068332

Scanga, C. A., Mohan, V. P., Joseph, H., Yu, K., Chan, J., and Flynn, J. L. (1999). Reactivation of latent tuberculosis: variations on the cornell murine model. Infect. Immun. 67, 4531-4538.

Singhal, A., Mathys, V., Kiass, M., Creusy, C., Delaire, B., Aliouat el, M., et al. (2011). BCG Induces Protection against Mycobacterium tuberculosis Infection in the Wistar rat model. PLOS ONE 6:e28082. doi: 10.1371/journal.pone.0028082

Wang, C. L., Pi, C. C., Kuo, C. W., Zhuang, Y. J., Khoo, K. H., Liu, W. H., et al. (2011). Polysaccharides purified from the submerged culture of Ganoderma formosanum stimulate macrophage activation and protect mice against Listeria monocytogenes infection. Biotechnol. Lett. 33, 2271-2278. doi: 10.1007/s10529-0110697-2

Woolhiser, L., Tamayo, M. H., Wang, B., Gruppo, V., Belisle, J. T., Lenaerts, A. J., et al. (2007). In vivo adaptation of the Wayne model of latent tuberculosis. Infect. Immun. 75, 2621-2625. doi: 10.1128/IAI.00918-06

Yeh, C. H., Chen, H. C., Yang, J. J., Chuang, W. I., and Sheu, F. (2010). Polysaccharides PS-G and protein LZ-8 from Reishi (Ganoderma lucidum) exhibit diverse functions in regulating murine macrophages and T lymphocytes. J. Agric. Food Chem. 58, 8535-8544. doi: 10.1021/jf1 $00914 \mathrm{~m}$

Yoshida, H., Suzuki, M., Sakaguchi, R., Tani, I., Kotani, H., Shudo, N., et al. (2012). Preferential induction of Th17 cells in vitro and in vivo by Fucogalactan from Ganoderma lucidum (Reishi). Biochem. Biophys. Res. Commun. 422, 174-180. doi: 10.1016/j.bbrc.2012.04.135

Zhang, T., Li, S.-Y., Williams, K. N., Andries, K., and Nuermberger, E. L. (2011). Short-course chemotherapy with TMC207 and Rifapentine in a murine model of latent tuberculosis infection. Am. J. Respir. Crit. Care Med. 184, 732-737. doi: 10.1164/rccm.201103-0397OC

Ziv, E., Daley, C. L., and Blower, S. M. (2001). Early therapy for latent tuberculosis infection. Am. J. Epidemiol. 153, 381-385. doi: 10.1093/aje/153.4.381

Conflict of Interest Statement: The authors declare that the research was conducted in the absence of any commercial or financial relationships that could be construed as a potential conflict of interest.

Copyright (c) 2016 Zhan, Tang, Lin, Xu, Xu and Qin. This is an open-access article distributed under the terms of the Creative Commons Attribution License (CC BY). The use, distribution or reproduction in other forums is permitted, provided the original author(s) or licensor are credited and that the original publication in this journal is cited, in accordance with accepted academic practice. No use, distribution or reproduction is permitted which does not comply with these terms. 